\title{
Revista Agrarian \\ ISSN: $1984-2538$ \\ Avaliação dos parâmetros físico-químicos e tecnológicos da farinha produzida a partir dos resíduos da agroindústria de laranja e melancia
}

\section{Evaluation of the physicochemical and technological parameters of flour produced from orange and watermelon agro-industry residues}

\author{
Antonio Carlos Pereira de Menezes Filho ${ }^{1}$, João Carlos Perbone de Souza ${ }^{1}$, Carlos Frederico de Souza \\ Castro ${ }^{1}$ \\ ${ }^{1}$ Instituto Federal Goiano Campus Rio Verde, Rod Sul-Goiana, km - 1, Zona Rural, CEP: 75.901-970, Rio \\ Verde-GO, Brasil. E-mail: astronomoamadorgoias@gmail.com
}

Recebido em: 12/12/2018

Aceito em:14/05/2019

\begin{abstract}
Resumo: As indústrias processadoras de frutas que movem o setor agroindustrial geram diariamente volumosos conteúdos de resíduos após processo de beneficiamento dos frutos. Tanto os frutos da laranja e da melancia são descartados em aterros sem nenhum processo de beneficiamento destes resíduos que podem gerar lucros anuais sobre estes coprodutos. Este trabalho teve como objetivo o aproveitamento dos resíduos (cascas e albedos) de laranjas e melancias para a produção de farinhas, sob este contexto avaliar alguns parâmetros físico-químicos e tecnológicos destas farinhas. Os resíduos foram coletados em indústrias processadoras de sucos no município de Rio Verde - GO, as farinhas foram preparadas por convecção térmica e analisadas para os seguintes parâmetros: $\mathrm{pH}$, molhabilidade (min.), atividade de água (aw), higroscopicidade ( $\mathrm{g} \mathrm{H}_{2} \mathrm{O}$ g MS), colorimetria $\mathrm{L}^{*} \mathrm{a}^{*} \mathrm{~b}^{*}$, chroma $C^{*}$, hue-angle $h^{\circ}$, índice de absorção de água e óleo (IAA/CAO), solubilidade em água (SA), volume de inchamento (VI) $\left(\mathrm{m}^{3} \mathrm{~kg}^{-1}\right)$, formação de espuma e gel, e por espectroscopia no infravermelho. Os resultados obtidos foram: $\mathrm{pH}, 4,07 ; 5,03$ / 3,31; 4,40, molhabilidade 75,$31 ; 84,89$ / 8,18; 10,31 (min.), aw 0,505; 0,489 / 0,490; 0,453, Higroscopicidade 0,16; 0,13 / 0,17; 0,20 g H2O g-1 MS, Cor L* 68,93; 72,86 / 84,92; 79,07, a* 4,52; -3,63 / 1,49; 3,50, b* 32,91; 24,62 / 18,$75 ; 18,00, C^{*} 18,81 ; 24,89 / 33,22 ; 18,34, h^{\circ} 85,46 ; 98,40 / 82,18 ; 79,00$, IAA 8,$68 ; 11,29 / 17,41$; 14,75\%, CAO 184,16; 321,19 / 244,99; 212,81\%, AS 13,14; 8,15 / 4,89; 23,00\%, VI 5,6; 6,9 / 14,33 13,13 $\mathrm{m}^{3} \mathrm{~kg}^{-1}$ respectivamente, para as farinhas das cascas e albedos de laranja e melancia. Não houve formação de espuma nos tempos (30 e 60 min.), formação de gel em todas as amostras na concentração mínima de 10\% e FT-IR apresentou espectros homogêneos com harmônicas entre as farinhas. As farinhas apresentaram boas características tanto físico-químicas quanto tecnológicas podendo ser empregadas em processos alimentícios.
\end{abstract}

Palavras-chave: FT-IR; atividade de água; higroscopicidade; colorimetria

\begin{abstract}
The fruit processing industries that move the agroindustrial sector, daily generate massive contents of residues after process of fruit processing. Both the orange and watermelon fruits are discarded in landfills without any processing of this waste that can generate annual profits on these co-products. The objective of this work was the use of the residues (shells and albedos) of orange and watermelon for the production of flours, in this context to evaluate some physicochemical and technological parameters of these flours. The residues were collected in juice processing industries in the municipality of Rio Verde - GO, the flours were prepared by thermal convection and analyzed for the following parameters: $\mathrm{pH}$, wettability (min.), water activity (aw), hygroscopicity (g H2O g $\left.{ }^{-1} \mathrm{MS}\right), \mathrm{L}^{*} \mathrm{a}^{*} \mathrm{~b}^{*}$ colorimetry, Chroma $\mathrm{C}^{*}$, hue-angle $\mathrm{h}^{\circ}$, water and oil absorption index (IAA / CAO), water solubility of swelling (VI) $\left(\mathrm{m}^{3} \mathrm{~kg}^{-1}\right)$, foaming and gel, and by infrared spectroscopy. The results were: $\mathrm{pH}, 4.07 ; 5.03 / 3.31 ; 4.40$, wettability $75.31 ; 84.89$ / 8.18; 10.31 (min), Aw 0.505; 0.489 / 0.490; 0.453, Hygroscopicity 0.16; 0.13 / 0.17; 0.20 g H2O g MS, Color L* 68.93; $72.86 / 84.92 ; 79.07, a^{*} 4.52 ;-3.63 / 1.49 ; 3.50, b^{*} 32.91 ; 24.62 / 18.75 ; 18.00$, C 18.81; $24.89 /$ 33.22 ; 18.34, h, 85.46; 98.40 / 82.18; 79.00, IAA 8.68; 11.29 / 17.41; 14.75\%, CAO 184.16; 321.19 / 244.99; $212.81 \%$, S $13.14 ; 8.15 / 4.89 ; 23.00 \%$, VI $5.6 ; 6,9 / 14,3313,13 \mathrm{~m}^{3} \mathrm{~kg}^{-1}$ respectively, for the cask meal and orange and watermelon albedos. There was no foaming times (30 and $60 \mathrm{~min}$ ), gel formation in all samples at the minimum concentration of $10 \%$ and FT-IR showed homogeneous spectra with harmonics between the flours. Flours have good physicochemical and technological characteristics can be used in food processes.
\end{abstract}

Keywords: FT-IR; water activity; hygroscopicity; colorimetry 


\section{Revista Agrarian}

ISSN: 1984-2538

\section{Introdução}

O uso de subprodutos obtidos a partir do processamento industrial gera grandes volumes de material orgânico que diariamente são descartados em aterros sanitários e lixões, sem qualquer tipo de uso como o reaproveitamento ou mesmo beneficiamento na geração de coprodutos como as farinhas. Ainda neste contexto, alguns autores ressalvam a utilização destes resíduos como forma econômica gerando lucros e a implementação de planos agroecológicos de manejo sustentável (Clemente et al., 2012; Alexandrino et al., 2007, Santana, 2005).

A utilização dos subprodutos é facilmente viável devido à grande produção industrial com nível constante de produção para o abastecimento do mercado nacional e internacional do beneficiamento das frutas, para produção de sucos concentrados, na extração de ácido cítrico e como sobremesas. O Brasil é o quarto maior produtor de melancia do mundo, com rendimento médio de 22 $\mathrm{t} \mathrm{ha}^{-1}$ ao ano avaliado para todos os estados brasileiros, entretanto, a região Nordeste apresenta os menores índices de rendimento médio de produção (Nascimento et al., 2017; Nascimento et al., 2015IBGE, 2015; Monção et al., 2012).

$\mathrm{Na}$ produção de laranjas o Brasil está entre os três maiores produtores mundiais, onde em 2016 a produção nacional foi de 17.251.291 t, com média de 26,180 t ha ${ }^{-1}$ (Coelho et al., 2019; IBGE, 2018). O estado de São Paulo é o primeiro maior produtor de laranja com média de 12.847.146 t, e o estado da Bahia o segundo maior produtor apresentando média anual de $1.129 .785 \mathrm{t}$ (Coelho et al., 2019; IBGE, 2018). Dados para a região noroeste do Estado do Paraná para o ano de 2010 obtiveram média de produção de 489 mil toneladas de laranjas, correspondendo a 25 toneladas por hectare de frutos colhidos, onde cerca de $96 \%$ da produção nacional de laranjas são direcionadas para o beneficiamento de suco. Esta grande produção, gera também grandes quantidades de resíduos que equivalem a $50 \%$ do peso bruto da fruta (Seab, 2013; Corazza et al., 2001).

Os resíduos gerados são pouco aproveitados, devendo ganhar a atenção quanto aos valores nutricionais presentes nos rejeitos das frutas. Estudos já comprovaram significativos conteúdos de fibras, complexos vitamínicos como A e C, proteínas, alto conteúdo de minerais,
Sódio, Potássio e Cálcio, bem como substâncias com características antioxidantes importantes que auxiliam na manutenção da saúde e combate os radicais livres como o oxigênio singlet (Menezes e Souza, 2018; Pereira et al., 2010; Clemente et al., 2007; Matsuura, 2005).

A laranja e melancia são frutos tipicamente apreciados pelo consumidor, ora na forma de sucos, frutas cristalizadas e geleias, estes frutos apresentam conteúdos fibrosos consideráveis conforme descrito por Clemente et al. (2012), os autores ainda complementam onde apresentam índices entorno de 80 milhões de toneladas ao ano na produção de laranjas. Além do uso das partes comestíveis destes frutos, a cultura brasileira ainda não aproveita os resíduos gerados nos próprios domicílios, podem ser desenvolvidos deliciosos doces com as cascas e o albedo dos frutos.

Os resíduos agroindustriais do processamento de laranja e melancia são as cascas (flavedo), bagaços (albedo), vesículas, sementes e aparas. Estas sobras podem-se ainda complementar a sua reutilização devido a ricas quantidades de componentes pécticos, celulose e alguns tipos de carboidratos hemicelulósicos (Clemente et al., 2012).

Como exemplo no desenvolvimento tecnológico da cadeia de produção alimentícia, temos a produção de farinhas para incorporação parcial ou total em alimentos como, pães, bolos, cookies, biscoitos, bolachas, mingaus e sopas (Farias Silva et al., 2016; Bender et al., 2016; Piovesana et al., 2013), iogurtes suplementados com farinhas (Curti et al., 2017), na texturização e como aditivo de fermentação de bebidas (GRAF et al., 2015), e in natura como condimentos alimentícios (Menezes e Souza, 2018).

$\mathrm{O}$ trabalho teve como objetivo o aproveitamento dos resíduos agroindustriais de laranja (Citrus sinensis L. Osbek, var. Pera-rio) e melancia (Citrullus lanatus Thumb. Mansf., var. Crimson Sweet Extra) após processo de extração do suco e da parte comestível das frutas, produzindo e avaliando os parâmetros físicoquímicos e funcionais tecnológicos das farinhas produzidas por convecção térmica.

\section{Material e Métodos}




\section{Revista Agrarian}

ISSN: 1984-2538

Os resíduos brutos agroindustriais foram sanitizados em solução clorada (1\%) por 15 minutos e lavados em água corrente. Logo em seguida, foram separados flavedo (casca) e albedo (parte branca) dos bagaços de laranja e melancia, descartando as sementes. Cada resíduo foi processado em liquidificador doméstico com 100 $\mathrm{mL}$ de água destilada. A massa foi filtrada em pano de nylon e distribuída em uma camada fina em formas de polietileno de alta densidadePEAD, onde foram levadas para secagem em estufa a $50{ }^{\circ} \mathrm{C}$ por 36 horas.

As farinhas foram produzidas utilizando moinho de facas tipo ciclone com peneira granulométrica interna com faixa 32 mesh. O "pó fino" produzido foi identificado por farinha flavedo laranja/melancia (FFL/FFM) e farinha albedo laranja/melancia (FAL/FAM), onde foram armazenadas em embalagens plásticas de polietileno para alimentos à $-8 \pm 1,0^{\circ} \mathrm{C}$ até serem analisadas.

$\mathrm{O} \mathrm{pH}$ foi avaliado conforme método descrito por IAL (1985) modificado, onde foi pesado $10 \mathrm{~g}$ de farinha em béquer de $150 \mathrm{~mL}$, logo após foi acrescido $100 \mathrm{~mL}$ de água destilada e deionizada. Esperou-se 15 minutos e leu-se utilizando pHmetro digital de bancada.

A molhabilidade foi determinada conforme descrito por Duarte et al. (2017), onde foi pesado $1 \mathrm{~g}$ de farinha e submetida a uma queda em frasco béquer $250 \mathrm{~mL}$ contendo $100 \mathrm{~mL}$ de água destilada e deionizada a $25 \pm 1,0{ }^{\circ} \mathrm{C}$, sem agitação e em bancada plana. Foi utilizado um cronômetro para determinar o tempo (em minutos (min.)) necessários para completa imersão $(100 \%)$.

Para atividade da água (aw) das farinhas armazenadas a temperatura ambiente, foi avaliada conforme descrito por Silva et al (2013), e Ferreira Neto et al. (2005) modificado, utilizando analisador de água HygroPalm, Modelo aw 1, acoplado a uma BOD com termostato controlado na temperatura de $25 \pm 1,0{ }^{\circ} \mathrm{C}$.

A higroscopicidade foi determinada conforme descrito por Silva et al. (2013), Tonon et al. (2009) modificado. Cerca de $1 \mathrm{~g}$ de farinha foi pesada em células de vidro boro, previamente seco a $105 \pm 2,0{ }^{\circ} \mathrm{C}$ por 3 horas em estufa com circulação e renovação de ar forçada. Logo após foram resfriados em dessecadora com sílica gel até temperatura ambiente de $25 \pm 1,0{ }^{\circ} \mathrm{C}$. As células foram levadas para um dessecador contendo atmosfera com $75 \%$ de umidade relativa, mantida por uma solução de cloreto de sódio PA., $40 \%$. As células permaneceram por 7 dias e a massa final foi pesada e a porcentagem de higroscopicidade determinada conforme Equação 1. Os resultados foram expressos em $\mathrm{g} \mathrm{H}_{2} \mathrm{O} \mathrm{g}{ }^{-1}$ MS.

Higroscopicidade $(\%)=(\mathrm{f}+\mathrm{H}) /(\mathrm{i}-\mathrm{H}) \times 100$

Onde: f, água adicionada; i massa inicial da amostra em (g); H, massa de água contida na amostra inicial em (g).

A cor foi avaliada utilizando colorímetro portátil. Os resultados foram expressos em L*, a*, $\mathrm{b}^{*}$, em que $\mathrm{L}^{*}$ (luminosidade ou brilho) variando de (0) preto ou (100) branco, croma a* (-60) verde e $(+60)$ vermelho e para croma $b^{*}(-60)$ azul ao $(+60)$ amarelo, conforme descrito por Dias e Leonel (2006). O Chroma $\left(C^{*}\right)$ foi determinado pela relação entre $\mathrm{a}^{*} \mathrm{e} \mathrm{b}^{*}$ conforme equação: $C^{*}$ $=\left[\left(\mathrm{a}^{*}\right)^{2}+\left(\mathrm{b}^{*}\right)^{2}\right]^{1 / 2}$, e o Hue-Angle $\left(h^{\circ}\right) h^{\circ}=\operatorname{arctg}$ $\mathrm{b}^{*} / \mathrm{a}^{*}$, sendo o ângulo formado entre $\mathrm{a}^{*} \mathrm{e} \mathrm{b}^{*}$, pela equação, $h^{\circ}=\tan ^{-1}\left(\mathrm{~b}^{*} / \mathrm{a}^{*}\right)$ indicando a saturação, descrito por Quelal-Vásconez et al. (2018) modificado.

O índice de absorção de água (IAA) foi determinado conforme descrito por Santana et al. (2017). Cerca de $0,5 \mathrm{~g}$ de farinha foi adicionada em $25 \mathrm{~mL}$ de água destilada, agitando-se manualmente por 1 minuto. Logo após, foi centrifugada a $3000 \mathrm{rpm}$ por 25 minutos. O líquido sobrenadante, foi utilizado par determinação da solubilidade em água (SA), foi escorrido e o material (farinha úmida) foi pesada. O líquido sobrenadante foi transferido para placa de Petri seca e levada para evaporar em estufa a $105^{\circ} \mathrm{C}$ com circulação e renovação de ar forçada.

A capacidade de absorção de em óleo (CAO) seguiu conforme metodologia descrita anteriormente, utilizando óleo de soja no lugar da água. O índice de absorção de água (IAA), Capacidade de absorção de óleo (CAO) e Solubilidade em água (SA) foram expressos conforme equações 2,3 e 4 .

$$
\begin{aligned}
& \mathrm{IAA}=\frac{\text { água absorvida pela amostra }(\mathrm{g})}{\text { peso da amostra }(\mathrm{g})} \\
& \mathrm{CAO}=\frac{\text { óleo absorvido pela amostra }(\mathrm{g})}{\text { amostra }(\mathrm{g})}
\end{aligned}
$$


$\mathrm{SA}=\underline{\text { resíduo de evaporação }(\mathrm{g})} \times 100$ peso da amostra $(\mathrm{g})$

O volume de inchamento foi determinado conforme descrita por Coffmann e Garcia (1977). Cerca de $1,0 \mathrm{~g}$ de farinha acrescida com $50 \mathrm{~mL}$ de água destilada foi agitada por 5 minutos em agitador magnético, logo em seguida, o volume foi transferido para uma proveta de $100 \mathrm{~mL}$ e calculado seu aumento percentual com base nos volumes inicial de acordo com a equação 5. A estabilidade da espuma foi avaliada ao deixar a proveta em repouso à temperatura ambiente 25 ${ }^{\circ} \mathrm{C}$, as aferições foram realizadas nos intervalos de 30 e 60 minutos. $\mathrm{O}$ volume de inchamento (VI) foi expresso conforme equação 5 .

$\mathrm{m}^{3} \mathrm{~kg}^{-1} \mathrm{VI}=(\mathrm{vf}-\mathrm{vi}) \times 100$

Onde: volume final (vf) e volume inicial (vi).

A capacidade de formação de gel da farinha foi determinada conforme descrito Santana et al. (2017). Para análise por espectroscopia no infravermelho com transformada de Fourier, foi utilizado equipamento com módulo ATR acoplado ao espectrômetro por infravermelho, onde a amostra da farinha foi comprimida sobre um cristal. Foram realizadas 5 varreduras em cada amostra, gerando os interferogramas.

Todas as análises foram realizadas em triplicata, exceto para colorimetria que foi realizada em quintuplicata. Os resultados foram submetidos à análise de variância e as médias seguidas de \pm desvio padrão, foram avaliadas pelo teste de Tukey $(\mathrm{p} \leq 0,05)$. Para análise estatística foi utilizado software Past3 (PAleontological Statistics, License: Freeware, 3.14).

\section{Resultados e Discussão}

$\mathrm{Na}$ Tabela 1, estão representados os resultados físico-químicos de $\mathrm{pH}$, tempo de molhabilidade, atividade de água e higroscopicidade das farinhas dos flavedos de laranja e melancia, e albedos de laranja e melancia.

Tabela 1. $\mathrm{pH}$, molhabilidade (min.), atividade de água (aw) e higroscopicidade \% $\left(\mathrm{g} \mathrm{H}_{2} \mathrm{O} / \mathrm{g}^{-1} \mathrm{MS}\right)$, nas farinhas do flavedo de laranja/melancia e albedo de laranja/melancia.

\begin{tabular}{lllll}
\hline Amostras & $\mathbf{p H}$ & $\begin{array}{l}\text { Molhabilidade } \\
(\mathbf{m i n} .)\end{array}$ & $\begin{array}{l}\text { Atividade de água } \\
\text { (aw) }\end{array}$ & $\begin{array}{l}\text { Higroscopicidade } \\
\left(\mathbf{g} \mathbf{~ H}_{2} \mathbf{O} \mathbf{g}^{-1} \mathbf{M S}\right)\end{array}$ \\
\cline { 2 - 5 } FFL & $4,07 \pm 0,01^{\mathrm{c}}$ & $75,31 \pm 2,00^{\mathrm{b}}$ & $0,505 \pm 0,00^{\mathrm{a}}$ & $0,16 \pm 0,10^{\mathrm{c}}$ \\
FFM & $5,03 \pm 0,10^{\mathrm{a}}$ & $84,89 \pm 1,40^{\mathrm{a}}$ & $0,489 \pm 0,00^{\mathrm{c}}$ & $0,13 \pm 0,41^{\mathrm{d}}$ \\
FAL & $3,31 \pm 0,10^{\mathrm{d}}$ & $8,18 \pm 0,14^{\mathrm{d}}$ & $0,490 \pm 0,00^{\mathrm{b}}$ & $0,17 \pm 0,10^{\mathrm{b}}$ \\
FAM & $4,40 \pm 0,00^{\mathrm{b}}$ & $10,31 \pm 0,00^{\mathrm{c}}$ & $0,453 \pm 0,00^{\mathrm{d}}$ & $0,20 \pm 0,12^{\mathrm{a}}$ \\
\hline
\end{tabular}

$\mathrm{pH}=$ realizado por potenciometria, Molhabilidade em minutos (min.) para molhar $100 \%$ da amostra, Atividade de água (Aw) e Higroscopicidade taxa de absorção de água em atmosfera controlada. MS = massa seca. Médias seguidas de \pm desvio padrão não diferem significativamente pelo teste de Tukey $(\mathrm{p} \leq 0,05)$

$\mathrm{O}$ pH mede a acidez, neutralidade ou alcalinidade em uma solução. As farinhas do flavedo apresentam $\mathrm{pH}$ entre 4,07 e 5,03 para laranja e melancia respectivamente. $\mathrm{E}$ entre as farinhas do albedo apresentando $\mathrm{pH}$ iguais a 3,31 e 4,40 para laranja e melancia respectivamente. Castro et. al. (2017) encontraram pHs entre 6,0 a 6,36 na farinha em três temperaturas e in natura de taro. Munhoz et al. (2010) avaliaram as farinhas da polpa e polpa com casca de goiabas desidratadas onde obtiveram $\mathrm{pH}$ de 4,28 e 4,29 respectivamente. Gomes et al. (2006) encontraram para farinha de feijão cru $\mathrm{pH}$ de 6,37 .

De acordo com Dzudie e Hardy (1996) o pH em suspensões de farinhas são importantes, já que algumas propriedades funcionais $\mathrm{e}$ tecnológicas são afetadas por alterações de $\mathrm{pH}$.

A molhabilidade neste estudo foi avaliada onde, $100 \%$ dos grânulos por minuto estariam completamente úmidos. A farinha do albedo de laranja apresentou menor tempo de 8,18 minutos e o maior tempo para a farinha do flavedo de melancia com 84,89 minutos. Duarte et al. (2017) avaliaram os pós dos frutos de marolo e cagaita onde obtiveram tempos de molhabilidade de 13,56 (0,23 min.) e 52,93 (0,88 min.). Lannes e Medeiros (2003) avaliaram como bom tempo em torno de 5 minutos para que $90 \%$ das partículas de cupuaçu em pó após spray-dryer se molharem em água. Todas as farinhas deste estudo apresentam 


\section{Revista Agrarian}

ISSN: 1984-2538

tempo em min. superiores ao preconizado por Lannes e Medeiros (2003), embora que este tempo máximo seja uma escolha arbitrária.

A molhabilidade é um parâmetro de tempo variável, aplicado a pós-alimentícios aos quais se deseja determinar o completo desaparecimento na superfície de um líquido, geralmente a água. Este parâmetro envolve propriedades físicas como tamanho da partícula e química envolvendo os teores de ácidos graxos conforme descrito por Duarte et al. (2017). Possivelmente a farinha da casca de melancia apresentam teores de ácidos graxos relativamente altos, dificultando que as partículas se molhem com facilidade devido a ação hidrofílica entre a água e as complexas cadeias carbônicas dos lipídeos.

A atividade de água (aw) nos fornece dados importantes sobre a umidade, observando que quanto mais baixo a atividade, mais lentas serão as reações bioquímicas e com isso menor mobilidade de atividade enzimática. A aw pode sofrer alterações conforme a umidade aumenta e quando a um tempo de secagem estabelecido, a Aw aumenta com a diminuição da temperatura e quando a temperatura é fixada, a aw diminui com o aumento de tempo para secagem (Silveira et al., 2016).

Neste estudo, a farinha do flavedo de melancia apresentou menor atividade de água com 0,489 aw e a maior atividade em farinha albedo laranja igual a 0,490 aw. Em estudo realizado por Capella (2008) onde avaliou e compararam as farinhas de pinhão-do-paraná em diferentes tratamentos, cru in natura, cru congelado, cozido in natura e cozido congelado obteve valores entre 0,351 a 0,819 aw respectivamente.
Duarte et al. (2017) avaliaram a aw nos frutos despolpados do marolo e cagaita in natura e liofilizados onde obtiveram médias de 0,250 e 0,300 aw respectivamente. Silveira et al. (2016) avaliaram o aproveitamento das sementes de goiaba para produção de farinha e encontraram resultado de 0,110 aw. Lima et al. (2015), obtiveram para a entrecasca in natura e farinha de melancia Aw iguais a 0,99 e 0,221 aw. Quando comparado ao deste estudo, a farinha do albedo de melancia apresentou atividade de água maior que o encontrado por Lima et al. (2015).

A higroscopicidade em alimentos desidratados principalmente farinhas, é um fator importante para a preservação e aumento de tempo de prateleira. Vieira Figueirêdo e Queiroz (2007), discuti sobre a influência do conteúdo de água sobre as variáveis, paladar, digestão estrutura física (formação de grumos) e manuseio (aumento de peso).

O conteúdo de água pode ser influenciado pelo tamanho da partícula, onde quanto maior a área superficial, maior será o contato com a água. Neste este estudo a higroscopicidade variou entre 0,13 a $0,20 \mathrm{~g} \mathrm{H}_{2} \mathrm{O} \cdot \mathrm{g}^{-1} \mathrm{MS}$, para as farinhas da casca melancia e albedo de melancia. Silva et al. (2013) encontraram para as farinhas de tapioca Santarém 0,58 e Santa Izabel $0,17 \mathrm{~g} \mathrm{H}_{2} \mathrm{O}_{\mathrm{g}} \mathrm{g}^{-1} \mathrm{MS}$.

Conforme Silva et al. (2013) valores altos de higroscopicidade, podem ser atribuídos a sua maior área superficial, indicando tratar de farinhas que requerem maiores cuidados no acondicionamento e armazenamento, estando mais susceptível ao ataque enzimático e por microrganismos, diminuindo a vida útil de prateleira do produto.

Tabela 2. Parâmetros colorimétricos, $\mathrm{L}^{*}$ luminosidade (0) preto ao (100) branco, croma a* (-60) verde ao (+60) vermelho, croma b* (-60) azul ou (+60) amarelo, Chroma $\mathrm{C}^{*}$ e Hue-Angle $h^{\circ}$ das farinhas do flavedo de laranja/melancia e albedo de laranja/melancia

\begin{tabular}{llllll}
\hline \multicolumn{2}{l}{ Cor } & \multicolumn{2}{l}{$\boldsymbol{C}^{*}$} & $\boldsymbol{h}^{\circ}$ \\
& $\mathbf{L}^{*}$ & $\mathbf{a}^{*}$ & $\mathbf{b}^{*}$ & & \\
FFL & $68,93 \pm 0,00^{\mathrm{d}}$ & $4,52 \pm 0,01^{\mathrm{a}}$ & $32,91 \pm 0,01^{\mathrm{a}}$ & $18,81 \pm 0,01^{\mathrm{c}}$ & $85,46 \pm 0,03^{\mathrm{b}}$ \\
FFM & $72,86 \pm 0,00^{\mathrm{c}}$ & $-3,63 \pm 0,01^{\mathrm{d}}$ & $24,62 \pm 0,01^{\mathrm{b}}$ & $24,89 \pm 0,01^{\mathrm{b}}$ & $98,40 \pm 0,04^{\mathrm{a}}$ \\
FAL & $84,92 \pm 0,01^{\mathrm{a}}$ & $1,49 \pm 0,01^{\mathrm{c}}$ & $18,75 \pm 0,01^{\mathrm{c}}$ & $33,22 \pm 0,01^{\mathrm{a}}$ & $82,18 \pm 0,01^{\mathrm{c}}$ \\
FAM & $79,07 \pm 0,00^{\mathrm{b}}$ & $3,50 \pm 0,00^{\mathrm{b}}$ & $18,00 \pm 0,01^{\mathrm{c}}$ & $18,34 \pm 0,01^{\mathrm{c}}$ & $79,00 \pm 0,02^{\mathrm{d}}$ \\
\hline
\end{tabular}

$\mathrm{FFL}=$ farinha flavedo laranja, $\mathrm{FFM}=$ farinha flavedo melancia, $\mathrm{FAL}=$ farinha albedo melancia e FAM $=$ farinha albedo melancia. Médias seguidas de \pm desvio padrão com mesma letra na coluna não diferem significativamente pelo teste de Tukey $(\mathrm{p} \leq 0,05)$

As farinhas apresentaram diferença significativa $(\mathrm{p} \leq 0,05)$ entre elas, para $\mathrm{L}^{*}$ variando entre 68,93 a 84,92 , o mesmo foi observado para croma a* entre $-3,63$ a 4,52 e para croma $b^{*} 24,62$ e 32,91 , exceto para as farinhas dos albedos de laranja e melancia que não

Menezes Filho et al., v.12, n.45, p. 399-410, Dourados, 2019 


\section{Revista Agrarian}

ISSN: 1984-2538

apresentaram diferença estatística, entre $\mathrm{b}^{*} 18,75$ e 18,00. A farinha do mesocarpo do pequi avaliado por Costa et al. (2017) apresentou L* de 73,70 , croma a* de 14,53 e croma $b^{*} 61,90$. As farinhas deste estudo possuem boa luminosidade, a farinha do flavedo de melancia possui $a^{*}$ tendendo para o verde e as demais amostras ao vermelho, a farinha do flavedo de laranja apresentou maior índice $b^{*}$ para cor amarela.

De acordo com Pathare et al. (2013) o componente chroma $\left(C^{*}\right)$ demonstra a intensidade da cor, onde quanto maior o resultado, maior será a intensidade da cor. O Hue Angle $\left(h^{\circ}\right)$ é representado por ângulos entre $0^{\circ}$ a $360^{\circ}$. Duarte et al. (2017) em seu estudo afirma que entre $0^{\circ}$ a $90^{\circ}$ a cor predominante é em tons de vermelho, laranja e amarelo, entre $90^{\circ}$ a $180^{\circ}$ tons de amarelo, amarelo esverdeado a verde, entre $180^{\circ}$ a $270^{\circ}$ tons de verde, azul esverdeado ao azul e entre $270^{\circ}$ a $360^{\circ}$ predominam tons do azul, púrpura, cores magentas e vermelhos.

Neste estudo pode-se observar a variação de $C^{*}$ entre 18,34 a 33,22 , estatisticamente as farinhas do flavedo de laranja e albedo de melancia apresentaram diferença significativa a ( $\mathrm{p}$ $\leq 0,05)$ e para $h^{\circ}$ de 79,00 a 98,40 houve significância a $(\mathrm{p} \leq 0,05)$ entre as farinhas dos flavedos e albedos de laranja e melancia.

Estudo realizado por Duarte et al. (2017) avaliando os frutos despolpados do marolo e cagaita in natura e liofilizados obtiveram $h^{\circ}$ de 86,63 e 84,84 e 89,50 e 88,72 respectivamente. Costa et al. (2017) encontraram para a farinha do mesocarpo do pequi $C^{*}$ de 61,95 e $h^{\circ}$ de 47,80 .

Tabela 3. Análise dos índices de absorção de água, capacidade de absorção em óleo, solubilidade em água, volume de inchamento, formação de espuma e formação de gel farináceo nas farinhas do flavedo de laranja/melancia e albedo de laranja/melancia

\begin{tabular}{lllll}
\hline Análises & FFL & FFM & FAL & FAM \\
\hline IAA (\%) & $8,68 \pm 0,51^{\mathrm{d}}$ & $11,29 \pm 0,52^{\mathrm{c}}$ & $17,41 \pm 0,45^{\mathrm{a}}$ & $14,75 \pm 0,73^{\mathrm{b}}$ \\
CAO (\%) & $184,16 \pm 10,82^{\mathrm{d}}$ & $321,19 \pm 16,02^{\mathrm{a}}$ & $244,99 \pm 7,99^{\mathrm{b}}$ & $212,81 \pm 3,62^{\mathrm{b}}$ \\
AS $(\%)$ & $13,14 \pm 0,57^{\mathrm{b}}$ & $8,15 \pm 0,03^{\mathrm{c}}$ & $4,89 \pm 0,70^{\mathrm{d}}$ & $23,00 \pm 0,90^{\mathrm{a}}$ \\
VI $\left(\mathbf{m}^{\mathbf{3}} \mathbf{k g}^{-\mathbf{1}}\right)$ & $5,6 \pm 0,16^{\mathrm{d}}$ & $6,9 \pm 0,08^{\mathrm{c}}$ & $14,33 \pm 0,31^{\mathrm{b}}$ & $13,13 \pm 0,30^{\mathrm{a}}$ \\
\hline Formação de Espuma & & & \\
\hline Tempo (min.) & FFL & FFM & FAL & FAM \\
\hline 30 & - & - & - & - \\
60 & - & - & - & - \\
\hline
\end{tabular}

Formação de Gel Farináceo

\begin{tabular}{llllllll}
\hline Amostras & $\mathbf{2 \%}$ & $\mathbf{6 \%}$ & $\mathbf{1 0 \%}$ & $\mathbf{1 4 \%}$ & $\mathbf{1 8 \%}$ & $\mathbf{2 2 \%}$ & $\mathbf{2 6 \%}$ \\
FFL & - & \pm & \pm & + & + & + & + \\
FFM & - & - & + & + & + & + & + \\
FAL & - & \pm & + & + & + & + & + \\
FAM & \pm & \pm & + & + & + & + & + \\
\hline
\end{tabular}

IAA \% = índice de absorção de água, $\mathrm{CAO} \%$ = capacidade de absorção de óleo vegetal, $\mathrm{AS} \%$ = resíduo de evaporação, $\mathrm{VI} \mathrm{m}^{3} \mathrm{~kg}^{-1}=$ volume de inchamento. $(-) /(+)$ formação de espuma. (-) sem formação de gel, $( \pm)$ gel frágil e $(+)$ gel resistente. Médias seguidas de \pm desvio padrão com mesma letra na mesma linha não diferem significativamente pelo teste de Tukey $(\mathrm{p} \leq 0,05)$

Costa et al. (2017) encontraram para a farinha do mesocarpo do pequi capacidade de absorção de 450,00\%. Neto (2012) também encontrou valor superior ao deste estudo para capacidade de absorção de água onde avaliou a farinha do mesocarpo de babaçu com taxa de absorção de água de $237,36 \%$. Borges et al. (2006) encontraram para farinha de trigo e três farinhas mistas $(15,30$ e $45 \%$ de aveia) valores de 53,$3 ; 58,3 ; 64,6$ e $72,0 \%$ de absorção de água. Estes valores são superiores aos observados neste estudo. De acordo com Neves et al. (2008), a hidratação do particulado revela diferenças estruturais de cada resíduo, afetando ou não a fibra no processo de absorção de água.

As farinhas das cascas e albedos de laranja e melancia apresentaram bom índice de absorção de óleo, com resultados de 184,16; 321,19 , e 244,99 e $212,81 \%$, sendo consideradas farinhas com boa qualidade para produtos viscosos como sopas e massas (Costa et al., 2017). Costa et al. (2017) demonstraram através de análise da capacidade de absorção de óleo utilizando a farinha do mesocarpo de pequi taxa de absorção igual a $173,33 \%$, valor próximo ao 


\section{Revista Agrarian}

ISSN: $1984-2538$

encontrado também por Neto (2012) para a farinha do mesocarpo do babaçu de $220,40 \%$.

A solubilidade em água (AS) entre as farinhas das cascas e albedos apresentaram diferença estatística $(\mathrm{p} \leq 0,05)$ entre elas, 13,14; 8,$15 ; 4,89$ e $23,00 \%$ respectivamente para laranja e melancia.

Santana et al. (2017) encontraram valores de $15,33 \%$ para SA na farinha de linhaça dourada, $10,00 \%$ para farinha de feijão branco e $10,00 \%$ para farinha de maracujá. Rios et al. (2018) encontraram médias para SA entre 17,86 a $22,40 \%$ para cinco farinhas integrais de cultivares comerciais de feijão variedade caupi. Conforme Leonel et al. (2009), farinhas que apresentam altos índices de solubilidade em água podem ser empregadas em misturas que necessitam de baixo aquecimento, também sendo utilizadas para sopas e molhos que necessitam de quantidades altas de água.

Quanto ao volume de inchamento (VI), neste estudo apresentou resultados para cascas de laranja e melancia e albedos laranja e melancia iguais a 5,$6 ; 6,9$ e 14,33 e $13,13 \mathrm{~m}^{3} \mathrm{~kg}^{-1}$ respectivamente. Resultados próximos foram obtidos por Garcia et al. (2017) onde avaliaram as farinhas das cascas e sementes do mamão, melão e goiaba, onde obtiveram volume de inchamento iguais a 3,$93 ; 8,67$ e 1,60 , e 3,$00 ; 4,20$ e $1,20 \mathrm{~m}^{3}$ $\mathrm{kg}^{-1}$ respectivamente. As farinhas dos albedos de laranja e melancia possuem alto poder de hidratação, sendo uma característica interessante para a indústria na produção de alimentos com consistência pastosa como mingaus (Garcia et al., 2017).

O VI é definido como o volume ocupado por uma amostra (farinha) conhecida, e a capacidade de retenção de água pelas fibras (Robertson et al., 2000). Os autores ainda complementam, que volumes até $20 \mathrm{~m}^{3} \mathrm{~kg}^{-1}$ são considerados como típicos para pós-fibrosos.

Neste estudo, as farinhas das cascas e albedos de laranja e melancia não apresentaram formação de espuma. O mesmo foi observado por Santana et al. (2017) para as farinhas de aveia A e $\mathrm{B}$, linhaça dourada e marrom e de uva. De acordo com os autores, e Porte et al. (2011), farinhas que não apresentam estas qualidades tecnológicas não podem ser utilizadas na elaboração de alimentos que requerem esta propriedade como no uso para fabricação de sorvetes e mousses.
A capacidade de formação de gel é utilizada como análise para avaliar a concentração mínima de farinha utilizada capaz de formar gel consistente em um determinado volume de água (Santana et al., 2017; Batista et al., 2010). A farinha do albedo de melancia em especial já apresentou gel frágil na menor concentração $2 \%$ seguida da farinha do albedo de laranja na concentração de 6\%. Quanto as farinhas dos flavedos, em especial a farinha do flavedo de laranja apresentou formação de gel frágil na concentração $6 \%$. A farinha do flavedo de melancia não apresentou formação de gel frágil, e sim, formação de gel resistente a partir da concentração de $10 \%$.

No trabalho realizado por Santana et al. (2017) utilizando farinhas comerciais de aveia A e B e trigo branca, os autores encontraram na concentração mínima de $2 \%$, formação de gel frágil como observado neste estudo para farinha do albedo de melancia. O mesmo não foi observado pelos autores na farinha de soja que não apresentou formação de gel em nenhuma das concentrações avaliadas.

Para Oro et al. (2013) e Jane et al. (1999) os produtos farináceos de origem vegetal podem conter entre 20 a $30 \%$ de amilose e de 70 a $80 \%$ de amilopectina, valores relativos entre os mais diversificados produtos, coprodutos e resíduos. Amidos com altas concentrações de amilopectina podem apresentar temperaturas de gelatinização mais altas outro fator importante é a viscosidade máxima que está relacionada ao comportamento dos grânulos de amido sobre a capacidade de absorção de água promovendo o inchamento durante ou não o processo térmico.

A Figura 1 abaixo apresenta os espectros por infravermelho com transformada de Fourier para as quatro farinhas dos albedos e cascas (flavedos) de laranja e melancia.

A espectroscopia de absorção na região média do infravermelho, foi utilizada para caracterizar as farinhas dos albedos e flavedos (cascas) dos frutos de melancia e laranja. Todas as amostras das farinhas analisadas apresentaram homogeneidade nas bandas dos espectros de FTIR como demonstrado na Figura 1, onde entre os comprimentos 3500 a $3000 \quad \mathrm{~cm}^{-1}$ são característicos do alongamento simétrico e assimétrico das ligações $\mathrm{O}-\mathrm{H}$. Neste estudo as bandas apresentaram em geral comprimentos em 3351,$7 ; 3333,0 ; 3330,5$ e $3294,4 \mathrm{~cm}^{-1}$ para as 


\section{Revista Agrarian}

ISSN: 1984-2538

farinhas da casca melancia, albedo laranja, albedo melancia e casca laranja, respectivamente. Mahecha (2009) encontrou para farinhas de biri (C. indica) bandas entre 3297 a $3283 \mathrm{~cm}^{-1}$.

$\mathrm{O}$ amido nativo neste estudo foi observado nas bandas em 2921,4, 2920,6; 2920,0 e $2917,2 \mathrm{~cm}^{-1}$ respectivamente para as farinhas, casca melancia, albedo laranja, albedo melancia e casca laranja, nesta faixa entre 3000 a $2900 \mathrm{~cm}^{-1}$ corresponde ao estiramento do grupo $\mathrm{C}-\mathrm{H}$. A presença deste alongamento foi reportada por Lima et al. (2012) que observaram bandas próximas ao deste estudo para farinhas de amido de milho, fécula de batata, banana-verde e trigo. Em 1606,8; 1605,8 e 1605,2 $\mathrm{cm}^{-1}$ para as farinhas da casca laranja, casca melancia e albedo de laranja, encontra-se a banda de amida I, vibrações de deformação axial de $\mathrm{C}=\mathrm{O}$, referente ao grupamento amida das proteínas.

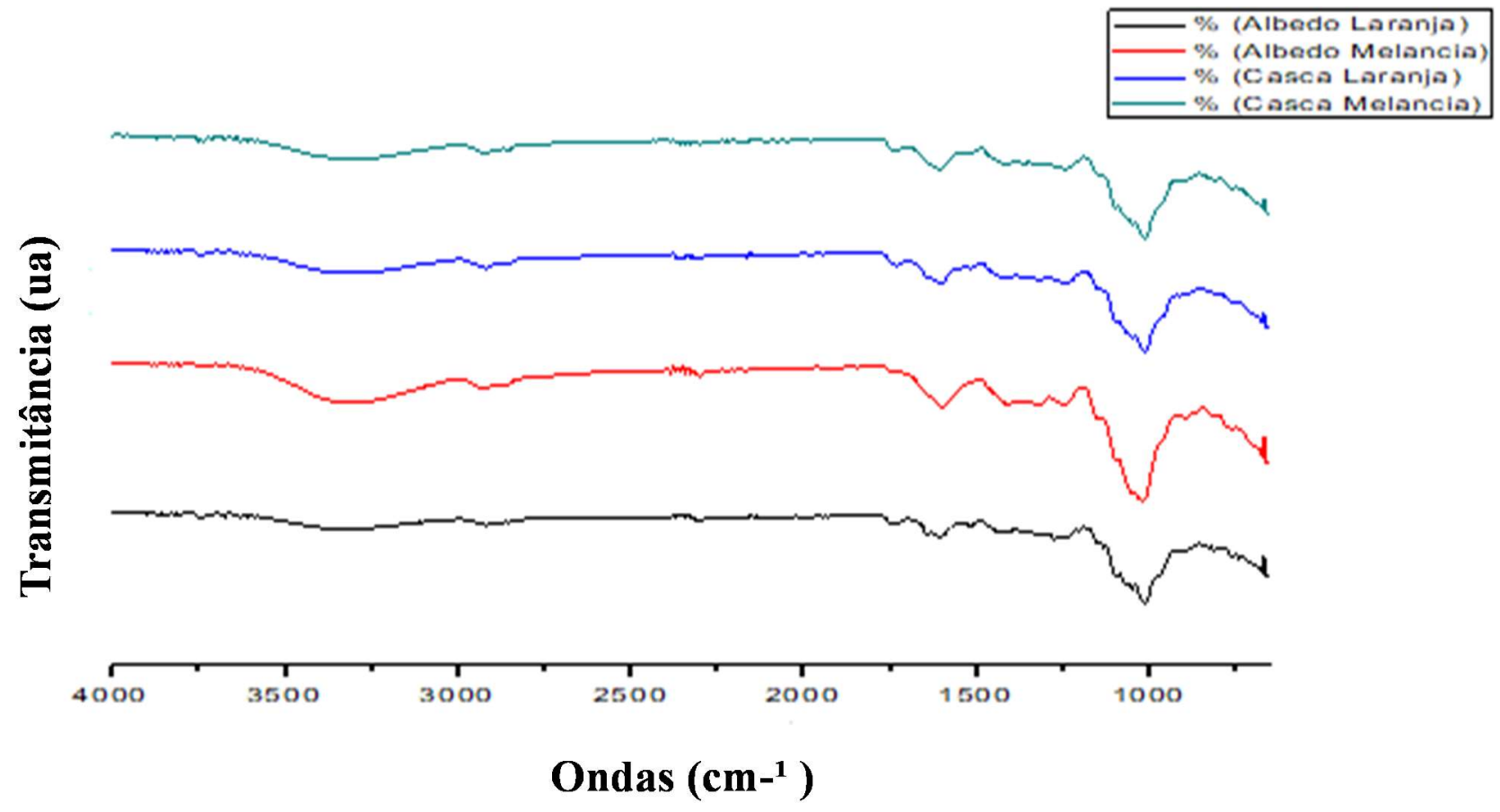

Figura 1. Análise por espectroscopia no infravermelho com transformada de Fourier (FT-IR), dos espectros das farinhas do albedo laranja e melancia, e flavedo (casca) laranja e melancia. Transmitância (u.a) e Ondas $\left(\mathrm{cm}^{-1}\right)$.

Em $1598,1 \mathrm{~cm}^{-1}$ na farinha do albedo de melancia, aparece uma banda importante referente a vibrações de deformação angular de N-H (banda amida II) entre 1620 a $1590 \mathrm{~cm}^{-1}$ (Silverstein et al., 2013). A banda em $1413,2 \mathrm{~cm}^{-1}$ na farinha do albedo de melancia, possivelmente está atribuída aos lipídeos (torção tipo tesoura) e proteínas. Dias (2008) encontrou uma banda em $1449 \mathrm{~cm}^{-1}$ avaliando farinhas e alguns dos seus componentes. Bandas em 1319,0; 1299,4; 1245,6 e $1242,4 \mathrm{~cm}^{-1}$ nas farinhas albedo melancia, albedo laranja, casca melancia e casca laranja respectivamente, correspondem a vibrações de deformação axial de C-O, deformação angular de $\mathrm{O}-\mathrm{H}$ e alongamento da ligação N-H e da flexão N$\mathrm{H}$ do grupo Amidas das proteínas. Dias (2008) caracterizando estruturalmente farinhas e alguns componentes farináceos encontrou uma banda em $1333 \mathrm{~cm}^{-1}$.

Bandas entre 1243,0;1095,5; 1051,0 e $1018,9 \mathrm{~cm}^{-1}$ das farinhas albedo melancia, albedo laranja, casca laranja e casca melancia respectivamente, são características do amido e atribuídas a vibrações de deformação axial dos grupos C-O, C-C e a vibrações de deformação axial do sistema O-C-O (Lima et al., 2012; Silverstein et al., 2013). Mahecha (2009) encontrou uma banda em $1016 \mathrm{~cm}^{-1}$, bandas próximas neste estudo sugere a presença de ligações $\mathrm{COH}$ ou solvatação, caraterística de materiais parcialmente cristalinos. Lima et al. 


\section{Revista Agrarian}

ISSN: 1984-2538

(2012) avaliando quatro farinhas obtiveram bandas entre 1077 a $928 \mathrm{~cm}^{-1}$.

Todos os espectros das quatro farinhas das cascas e albedos de laranja e melancia apresentaram harmônicas entre 2400 a $1900 \mathrm{~cm}^{-1}$. Nesta análise é possível verificar a homogeneidade das amostras e os grupos funcionais característicos observados nas farinhas deste estudo, e quando comparados ao das literaturas analisadas.

\section{Conclusão}

As farinhas dos flavedos e albedos de laranja e melancia apresentam boas características funcionais tecnológicas para serem introduzidas em nos mais variados tipos de alimentos funcionais. $\mathrm{O}$ uso de resíduos agroindustriais necessita ser amplamente avaliado, dando destino como o aproveitamento deste material que até então, seriam descartados em aterros sanitários ou lixões a céu aberto gerando volumosas quantidades de material orgânico.

\section{Agradecimentos}

Ao Instituto Federal de Educação, Ciência

e Tecnologia Goiano, Campus Rio Verde; aos Laboratórios de Química Tecnológica, Sementes e Pós-Colheita, e de Eletroanalítica; a Central Multiusuário Analítico CEMA; a CAPES, CNPq, FINEP e FAPEG, esta última pela bolsa de mestrado para o primeiro autor Antonio.

\section{Referências}

ALEXANDRINO, A. M.; FARIA, H. G.; SOUZA, C. G. M.; PERALTA, R. M. Aproveitamento do resíduo de laranja para a produção de enzimas lignino-celulósicas por Pleurotus ostratus (Jack: Fr.). Food Science and Technology, v. 27, n. 2, p. 364-368, 2007.

BENDER, A. B. B.; LUVIELMO, M. de M.; LOUREIRO, B. B.; SPERONI, C. S.; BOLIGON, A. A.; SILVA, L. P da.; PENNA, N. G. Obtenção e caracterização de farinha de casca de uva e sua utilização em snack extrusado. Brazilian Journal of Food Technology, v. 19, n. e2016010, p. 1-9, 2016.

BORGES, J. T. da S.; PIROZI, M. R.; LUCIA, S. M. D.; PEREIRA, P. C.; MORAES, A. R. F.; CASTRO, V. C. Utilização de farinha mista de aveia e trigo na elaboração de bolos. Boletim CEPPA, v, 24, n. 1, p. 145-162, 2006.

CAMARGO FILHO, W. P.; MAZZEI, A. R. O mercado de melancia no Mercosul. Revista Informações Econômicas, v. 32, n. 2, p. 61-64, 2002.

CASTRO, D. S de.; OLIVEIRA, T. K. B de.; LEMOS, D. M.; ROCHA, A. P. T.; ALMEIDA, R. D. Efeito da temperatura sobre a composição físico-química e compostos bioativos de farinha de taro obtida em leito de jorro. Brazilian Journal of Food Technology, v. 20, p. 1-5, 2017.

CHABARIBERY, D.; ALVES, H. S. Produção e comercialização de limão, mamão, maracujá e melancia em São Paulo. Revista Informações Econômicas, v. 31, n. 8, p. 43-51, 2001.

CLEMENTE, E.; FLORES, A. C.; ROSA, C. I. L. F.; OLIVEIRA, D. M. Características da farinha de resíduos do processamento de laranja. Revista Ciências Exatas e Naturais, v. 14, n. 2, p. 257269, 2012.

COELHO, B. E. S.; DUARTE, V. M.; SILVA, L. F. M. da.; SOUSA, K. dos. S. M. de.; NETO, A. F. Atributos físico-químicos de frutos de laranja 'Pêra' produzidos sob sistemas de cultivo orgânico e convencional. Revista Brasileira de Meio Ambiente, v. 5, n. 1, p. 128-137, 2019.

COFFMANN, C. N.; GARCIA, V. V. Functional properties and amino acid content of a protein isolate from mung bean flour. International Journal of Food Science and Technology, v. 12, n. 5 , p. $473,1977$.

CORAZZA, M.; RODRIGUES, D. G.; NOZAKI, J. Preparação e caracterização do vinho de laranja. Revista Química Nova, v. 24, n. 4, p. 449-452, 2001.

COSTA, A. P. F da.; PINTO, E. G.; SOARES, D. S. B. Obtenção de farinha do mesocarpo de pequi. Revista Agrarian, v. 10, n. 38, p. 349-354, 2017.

CURTI, C. A.; VIDAL, P. M.; CURTI, R. N.; RAMÓN, A. N. Chemical characterization, texture and consumer acceptability of yorgurts supplemented with quinoa flour. Food Science and Technology, v. 37, n. 4, p. 627-631, 2017. 


\section{Revista Agrarian}

ISSN: 1984-2538

DIAS, A. M. V. Análise estrutural de farinhas e alguns dos seus componentes. 2008. Ano de obtenção 2008. 148 f. Dissertação (Mestrado em Química e qualidade dos alimentos), Universidade de Aveiro, Portugal, 2008.

DIAS, L. T.; LEONEL, M. Caracterização físicoquímica de farinhas de mandioca de diferentes localidades do Brasil. Revista Ciência Agrotecnologia, v. 30, n. 4, p. 692-700, 2006.

DUARTE, E. L.; CARLOS, L. de A.; RODRIGUES, C. G.; ANDRADE, R. M.; OLIVEIRA, K. G de. Influência da liofilização sobre os carotenoides de frutos do cerrado e comportamento hidroscópico dos produtos liofilizados. Revista Perspectivas Online: Biologia \& Saúde, v. 23, n. 7, p. 22-33, 2017.

DZUDIE, T.; HARDY, J. Physicochemical and functional properties of flours prepared from common beans and green mung beans. Journal of Agriculture and Food Chemistry, v. 44, p. 3029-3032, 1996.

FAO (Roma, Italy). Agricultural production, primary Crops. Disponível em http: www.fao.org. Acesso em: 29/11/ 2018.

GARCIA, D. M.; ALENCAR, U. R de.; MOTA, B. G.; BORGES, I. R.; SOUZA, P. O. Determinação de características tecnológicas de farinhas produzidas a partir de resíduos de polpas de mamão, melão e goiaba e sua utilização na elaboração de biscoito tipo cookies. ScientiaTec: Revista de Educação, Ciência e Tecnologia do IFRS, v. 4, n. 1, p. 29-41, 2017.

GRAF, B. L.; ROJAS-SILVA, P.; ROJO, L. E.; DELATORRE-HERRERA, J.; BALDEÓN, M. E.; RASKIN, I. Innovations in health value and functional food development of Quinoa (Chenopodium quinoa Willd.). Comprehensive Reviews in Food Science and Food Safety, v. 14, n. 4, p. 431-445, 2015.

GRANGEIRO, L. C.; CECÍlIO FILHO, A. B. Exportação de nutrientes pelos frutos de melancia em função de épocas de cultivo, fontes e doses de potássio. Revista de Horticultura Brasileira, v. 22, n. 4, p. 740-743, 2004.
IBGE (2015) Instituto Brasileiro de Geografia e Estatística, Levantamento Sistemático da Produção Agrícola - LSPA. Disponível em http://www.sidra.ibge.gov.br/bda/agric. Acesso em: 25/09/2019.

IBGE (2018) Instituto Brasileiro de Geografia e Estatística, Levantamento Sistemático da Produção Agrícola - LSPA. Disponível em http:/www.ibge.gov.br/estatísticas-

novoportal/econômicas/agricultura-e-pecuaria.

Acesso em: 25/09/2018.

LANNES, S. C da S.; MEDEIROS, M. L. Processamento de achocolatado de cupuaçu por spray-dryer. Revista Brasileira de Ciências Farmacêuticas, v. 39, n. 1, p. 115-123, 2003.

LIMA, B. N. B.; CABRAL, T. B.; NETO, R. P. C.; TAVARES, M. I. B.; PIERUCCI, A. P. T. Estudo do amido de farinhas comerciais comestíveis. Revista Polímeros, v.22, n. 5, p. 486-490, 2012.

MAHECHA, M. M. A. Elaboração e caracterização de biofilmes de farinha de biri (Canna indica L.). 2009. Ano de obtenção: 2009. 159 f. Dissertação (Mestrado em Engenharia de Alimentos), Universidade Federal de Campinas, 2009.

MATSUURA, F. C. A. U. Estudo do albedo de maracujá e de seu aproveitamento em barra de cereais. 2005. Ano de obtenção: 2005. 138 f. Tese (Doutorado em Engenharia de Alimentos), Universidade Estadual de Campinas, Campinas, 2005.

MENEZES, A. C. P. de.; SOUZA, C. F. de. C. Aproveitamento dos resíduos de melancia na produção de condimento para uso em alimentos. Revista Informe Goiano, v. 03, n. 08, p. 1-4, 2018.

MONÇÃO, O. P.; RIBEIRO, J. J.; MOSCON, E. S.; OLIVEIRA, D. N. S.; NASCIMENTO NETO, J. G. Produtividade da cultura da melancia sob diferentes doses de potássio no município de Santa Rita de Cássia - BA. Enciclopédia Biosfera, v. 8, n. 15, p. 1423-1424, 2012. 


\section{Revista Agrarian}

ISSN: 1984-2538

MUNHOZ, C. L.; SANJINEZ-ARGANDOÑA, E. J.; SOARES-JÚNIOR, M. S. Extração de pectin de goiaba desidratada. Revista Ciência e Tecnologia de Alimentos, v. 30, n. 1, p. 119-125, 2010.

NASCIMENTO, J. A. M.; SOUTO, J. S.; CAVALCANTE, L. F.; OLIVEIRA, F. T.; MENDONÇA, V.; ALBUQUERQUE JÚNIOR, A. M.; MEDEIROS, S. A. S. Macronutrientes na cultura da melancia cultivada em Neossolo com esterco bovino. Revista Brasileira de Ciências Agrárias, v. 10, n. 2, p. 224-229, 2015.

NASCIMENTO, J. A. M. do.; SOUTO, J. S.; CAVALCANTE, L. F.; MEDEIROS, S. A. da. S.; PEREIRA, W. E. Produção de melancia em solo adubado com esterco bovino e potássio. Agrária - Revista Brasileira de Ciências Agrárias, v. 12, n. 2, p. 122-127, 2017.

NETO, A. A. C. Desenvolvimento de massa alimentícia mista de farinhas de trigo e mesocarpo de babaçu (Orbignya sp.). 2012. Ano de obtenção: 2012. 68 f. Dissertação (Mestrado em Ciência e Tecnologia de Alimentos). Universidade Federal Rural do Rio de Janeiro, Seropédica, 2012.

ORO, T.; LIMBERGER, V. M.; MIRANDA, M. Z.; RICHARDS, N. S. P. S.; GUTKOSKIL, L. C.; FRANCISCO, A. Propriedades de pasta de mesclas de farinha integral com farinha refinada usadas na produção de pães. Revista Ciência Rural, v. 43, n. 4, p. 754-760, 2013.

PEREIRA, A. S.; MIGUEL, D. P.; CARVALHO, E. E. N. Caracterização de farinha de entrecasca de melancia (Citrullus tanatus) produzida na região sul do Tocantins. Cadernos de PósGraduação da FAZU, n. 1, p. 1-5, 2010.

PIOVESANA, A.; BUENO, M. M.; KLAJN, V. M. Elaboração e aceitabilidade de biscoitos enriquecidos com aveia e farinha de bagaço de uva. Brazilian Journal of Food Technology, v. 16, n. 1, p. 68-72, 2013.

PORTE, A.; SILVA, E. F.; ALMEIDA, V. D. S.; SILVA, T. X.; PORTE, L. H. M. Propriedades funcionais tecnológicas das farinhas de sementes de mamão (Carica papaya) e de abóbora
(Curcubita sp.). Revista Brasileira de Produtos Agroindustriais, v. 13, n. 1, p. 91-96, 2011.

RIOS, M. J. B. L.; DAMASCENO-SILVA, K. J.; MOREIRA-ARAÚJO, R. S. dos R.; FIGUEIREDO, E. A. T de.; ROCHA, M. de M.; HASHIMOTO, J. M. Chemical, granulometric and technological characteristics of whole flours from comercial cultivars of cowpea. Revista Caatinga, v. 31, n. 1, p. 217-224, 2018.

ROBERTSON, J.; MONREDON, F. D.; DYSSELER, P.; GUILLON, F.; AMADO, R.; HIBAULT, J. F. Hydration properties of dietary fibre and resistant starch: a European collaborative study. Lebensmittel-Wissenschaft und Technologie, v. 33, p. 72-79, 2000.

SANTANA, G. S.; OLIVEIRA FILHO, J. G de.; EGEA, M. B. Características tecnológicas de farinhas vegetais comerciais. Journal of Neotropical Agriculture Neotropical, v. 4, n. 2, p. 88-95, 2017.

SANTANA, M. F. S. Caracterização físicoquímica de fibra alimentar de laranja e maracujá. 2005. Ano de obtenção: 2005. 168 f. Tese (Doutorado em Engenharia de Alimentos), Universidade Estadual de Campinas, Campinas, 2005.

SEAB. Secretaria da Agricultura e do Abastecimento. DERAL. Departamento de Economia Rural. Produção Agropecuária. 2011. Disponível em http://www.agricultura.pr.gov.br/modules/conteud o/conteudo.php?conteudo=137. Acesso em: 14/07/2018.

FARIAS SILVA, C. E. de.; GAMA, B. M. V. da.; OLIVEIRA, L. M. T. de.; ARAÚJO, L. T. de.; ARAÚJO, M. L. de.; OLIVEIRA JÚNIOR, A. M.; ABUD, A. K. de. S. Uso da laranja lima e seus resíduos no desenvolvimento de novos produtos. Brazilian Journal of Biosystems Engineering, v. 10, n. 1, p. 69-96, 2016.

SILVERSTEIN, R. M.; WEBSTER, F. X.; KIEMLE, D. J. Identificação espectrométrica de compostos orgânicos. $7^{\text {a }}$ Ed., Ed. LTC, 2013, p. 490. 


\section{(2) \\ Revista Agrarian \\ ISSN: $1984-2538$}

SILVIA, P. A.; CUNHA, R. L.; LOPES, A. S.; PENA, R. da S. Caracterização de farinhas de tapioca produzidas no estado do Pará. Revista Ciência Rural, v. 43, n. 1, p. 185-191, 2013.

QUELAL-VÁSCONEZ, M. A.; PÉREZESTEVE, É.; ARNAU-BONACHERA, A.; BARAT, J. M.; TALENS, P. Rapid fraud detection of cocoa powder with carob flour using near infrared spectroscopy. Food Control, v. 92, p. 183-189, 2018. 